\title{
APLICACIÓN DEL ANÁLISIS ESTADÍSTICO PARA LA HOMOGENEIZACIÓN CLIMATOLÓGICA E HIDROLÓGICA EN LA CUENCA DEL RÍO ARAGÓN AGUAS ARRIBA DEL PANTANO DE YESA (PIRINEO CENTRAL)
}

\author{
Ahmed ELMOUJAHID \\ Departamento de Geografía y Ordenación del Territorio \\ Universidad de Zaragoza
}

\begin{abstract}
Resumen: Se expone la aplicación del método de análisis de frecuencia regional, con el objetivo de identificar una subcuenca climática e hidrológicamente homogenea. Se han utilizado series completas de datos de precipitaciones y caudales representando 30 estaciones climáticas y 8 estaciones de aforo.
\end{abstract}

La variabilidad del coeficiente de variación (Cv) y del coeficiente de sesgo (Cs) de las precipitaciones medias anuales (PMA) y la localización geográfica han permitido la identificación de tres regiones climáticamente homogéneas ( $\mathrm{RCH}$ ). También se identificaron las subcuencas hidrológicamente homogéneas ( $\mathrm{ScHH}$ ), analizando los $\mathrm{Cv}$ y $\mathrm{Cs}$ de los caudales máximos de medias diarias de las estaciones de aforo y se ha aplicado el test de homogeneidad de Gumbel. A partir de ambas identificaciones se ha logrado especificar la subcuenca climática e hidrológicamente homogenea $(\mathrm{ScCH})$. Por último, se formulan comentarios relativos a los resultados, destacando la utilidad del metodo de análisis estadístico en la homogeneización climática e hidrológica.

Palabras clave: análisis de frecuencia regional, homogeneidad, test de homogeneidad de Gumbel, río Aragón.

\begin{abstract}
A regional frequency analysis procedure is applied to identify a climatologically and hydrologically homogeneous catchment. A complete data sets of precipitation and discharge representing 30 meteorological stations and 8 of hydrometric stations were used for various statistical analyses.

Variability of the coeficient of variation $(\mathrm{CV})$ and the coeficient of skewness (Cs) and the mean annual precipitation (MAP) and geographical locations has been explained to identify three climatologically homogeneous regions (CHR). Also the analysis of $\mathrm{Cv}$ and $\mathrm{Cs}$ of anual maximum of daily meam discharge were examined to identify hydrologically homogeneous catchments ( $\mathrm{HHC}$ ) and application of procedure Gumbel test of homogeneity. Based on results of both identifications a climatologically and hydrologically homogeneous catchment has been specified.
\end{abstract}


Finally some remarks are presented concerning the usual statistics analysis method and some conclusions are drown for these particular example work.

Key words: Analysis of Regional Frecuency, Homogeneity Gumbel test, Aragon river.

\section{INTRODUCCIÓN}

Recientemente se ha emprendido un gran esfuerzo en temas de análisis de Ia frecuencia regional, en los que se utilizan datos de las estaciones climáticas que se encuentran en una región homogénea. Varias técnicas de frecuencia regional han sido propuestas por los investigadores, y entre ellas:

- Aplicación de la estadística del coeficiente de variación (Cv) cuando se analiza la precipitación extrema para una duración particular, porque visualiza distintos patrones geográficos que sobrepasan ampliamente las fluctuaciones de precipitación.

- Procedimiento de análisis regional utilizando las relaciones de los momentos lineares (momentos-L) con dos curvas de regresión regional del coeficiente de variación (Cv) y del coeficiente de asimetría (Cs), y la precipitación media anual (PMA).

- Esquema de regionalización para regiones con una alta variabilidad del CV, del Cs y largas variaciones de precipitación media anual (PMA), utilizando el Cv y el Cs como variables y desarrollando dos curvas de regresión para explicar las relaciones entre Cv y la PMA, así como entre Cs y la PMA.

El procedimiento de análisis regional es, en realidad, una combinación del examen de las características físicas y el análisis de los parametros estadisticos (CEDEX, 1994). Pero la dificultad que supone el estudio más detallado del aspecto geográfico, impone que se puede adoptar únicamente el análisis de los parámetros estadísticos, para una homogeneización relativa en función de los elementos de representación proyectada.

Ferrer y Ardiles (CEDEX, 1994) han utilizado las series anuales de máximas díarias a escala nacional (España) de 1545 estaciones pluviométricas con más de 30) años de registros, que han sido agrupadas en 26 regiones delimitadas con criterios meteorológicos y cuya homogeneización ha sido contrastada mediante análisis estadístico de los coeficientes de variación muestrales.

FANG Y NAGHAVI (1995) consideran que la variabilidad del clima de un sitio a otro influye ampliamente sobre el esquema de regionalización, y quizás es preferible 
subdividir la región en pequeñas subregiones utilizando otro criterio que el $\mathrm{Cv}$. Tal futura subdivisión puede ser cumplida usando la precipitación media anual y la ubicación geográfica de las estaciones climatológicas. Procedimiento que parece más aséquible y que se puede emprender en la zona del alto Aragón (aguas arriba del pantano de Yesa) en el Pirineo central.

\section{METODOLOGÍA}

Se propone llevar a cabo un análisis estadístico, con los siguientes objetivos:

1- Formulación de un procedimiento práctico de análisis regional y su aplicación. a los datos de las precipitaciones y caudales de la zona de estudio.

2-Teniendo en cuenta la complejidad de las características geográficas del área de estudio, y con el fin de reducir más posible las variables, se propone llevar a cabo el estudio de homogeneización para identificar la subcuenca climática e hidrológicamente homogénea representativa de la zona de estudio, utilizando las series completas de datos (1950-1995) representando las 30 estaciones climáticas y las 8 estaciones de aforo.

El método de análisis de frecuencia regional de la zona de estudio consiste en las siguientes etapas:

a- Identificación, primero, de las Regiones Climatológicamente Homogéneas $(\mathrm{RCH})$ usando la precipitación media anual y la ubicación geográfica de las estaciones climatológicas.

b- Análisis de la variabilidad del Cv y del Cs determinados a partir de las series de precipitación anual y la precipitación máxima de 24 horas dentro de las $\mathrm{RCH}$.

c- Identificación de las Subcuencas Hidrológicamente Homogéneas ( $\mathrm{ScHH}$ ), usando también en primer lugar la aportación media anual y los caudales máximos diarios de las estaciones de aforo.

d- Análisis de la variabilidad del $\mathrm{Cv}_{\mathrm{v}}$ y del Cs de los datos de caudales máximos díarios de las estaciones de aforo.

e- Aplicación del test de homogeneidad según la ley de Gumbel para probar cuál de las estaciones de aforo puede considerarse formando una $\mathrm{ScHH}$. 


\section{SITUACIÓN DE LA ZONA DE ESTUDIO}

La zona elegida para la realización de este estudio coincide con la cuenca hidrográfica del río Aragón aguas arriba del pantano de Yesa, perteneciendo su superficie a las provincias de Zaragoza, Huesca y Navarra. En el punto de ubicación de la estación de aforo del embalse de Yesa, la cuenca vertiente tiene una superficie de $2191 \mathrm{~km}^{2}$ y está dividida por las subcuencas de los ríos Esca, Veral, Subordán y la cabecera del río Aragón (Fig.1).

Limita al Norte con Francia, en pleno Pirineo axil, al Este con la cuenca del río Gállego, al Oeste con la cuenca del río Salazar y al Sur con las Sierras Exteriores. Las altitudes en la cuenca oscilan desde las cotas $500 \mathrm{~m}$ en la E-829 en el punto de ubicación de la presa, hasta la cota $2886 \mathrm{~m}$ en su cabecera (pico Collarada).

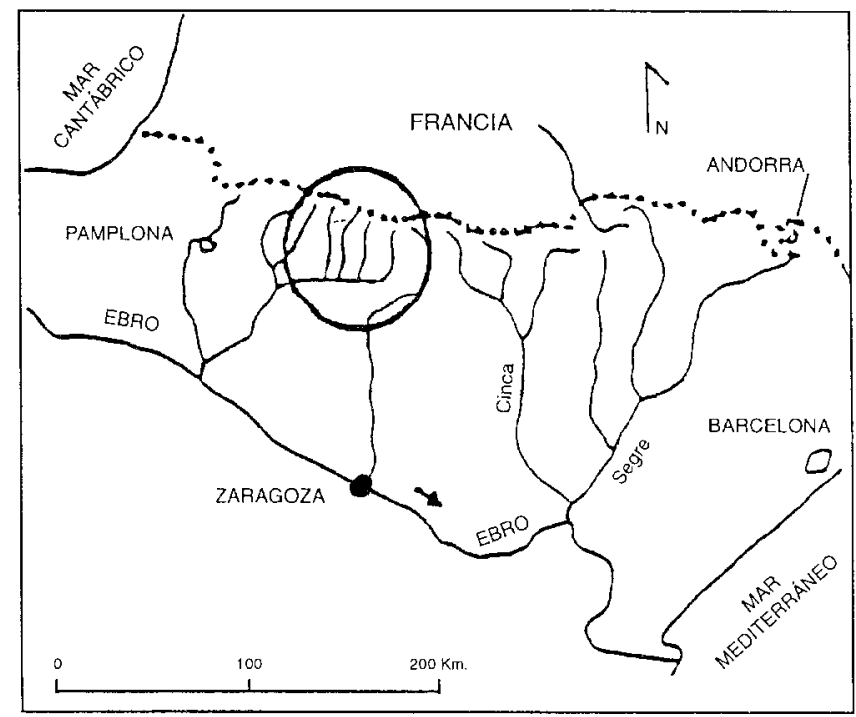

Figura 1.- Localización del área de estudio. 


\section{INFORMACIÓN CLIMÁTICA E HIDROMÉTRICA UTILIZADA}

Los datos de precipitación utilizados en la realización de este estudio han sido obtenidos del banco de datos del Instituto Meteorológico Nacional de Madrid, mientras que los datos hidrométricos de las 8 estaciones de aforo provienen de los anuarios de registros de la Confederación Hidrográfica del Ebro. En cuanto al período temporal, se han tomado las series isocrónicas de cuarenta y cinco años del periodo 1950 - 1995, correspodientes a los siguientes datos:

- Precipitaciones medias anuales y diarias máximas anuales.

- Aportaciones anuales y caudales diarios máximos anuales.

\section{IDENTIFICACIÓN DE LAS REGIONES CLIMÁTICAMENTE HOMOGÉNEAS (RCH)}

\section{Análisis de los parámetros estadísticos}

Para las RCH los valores del coeficiente de variación (Cv) y el coeficiente de asimetría (Cs) en una importante población regional se suponen constantes (FANG Y NAGHAVI, 1995). Entonces, y basándonos en esta consideración, los resultados de los gráficos de la precipitación media anual (Gráfico 1), Cv (Gráfico 2) y Cs (Gráfico 3), aconsejan que la zona de estudio esté dividida en tres regiones climatológicamente homogéneas, que corresponden a la región norte del Pirineo Axial (I), región central de las Sierras Interiores (II) y la región de la Canal de Verdun (III) (Fig. 2). La tabla 1 resume los resultados.

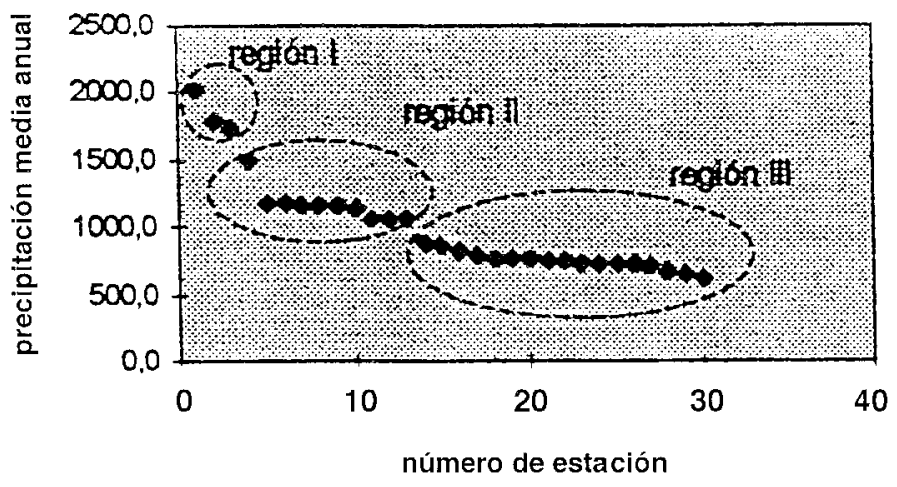

Grafico 1.- Identificación de regiones homogéneas. 


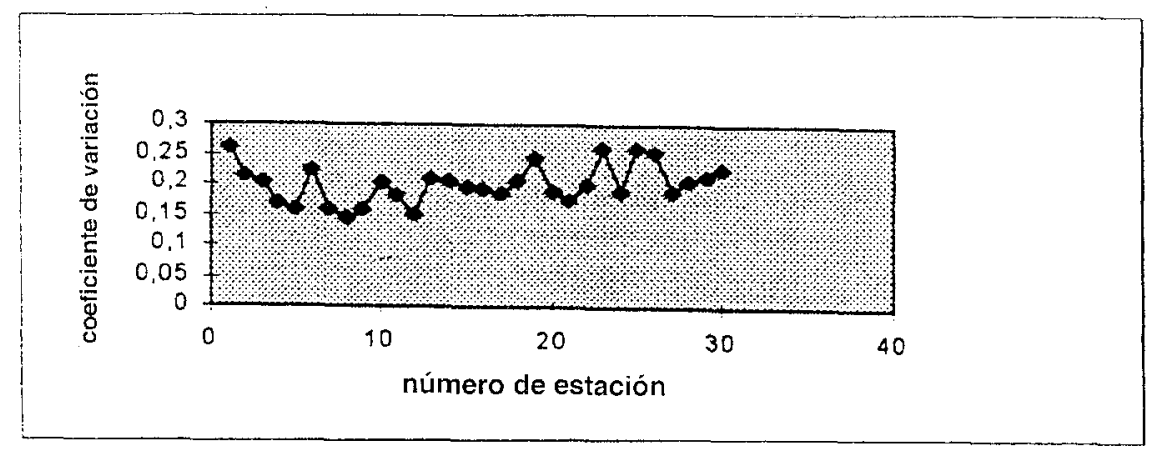

Grafico 2.- Curva de variación del Cv.

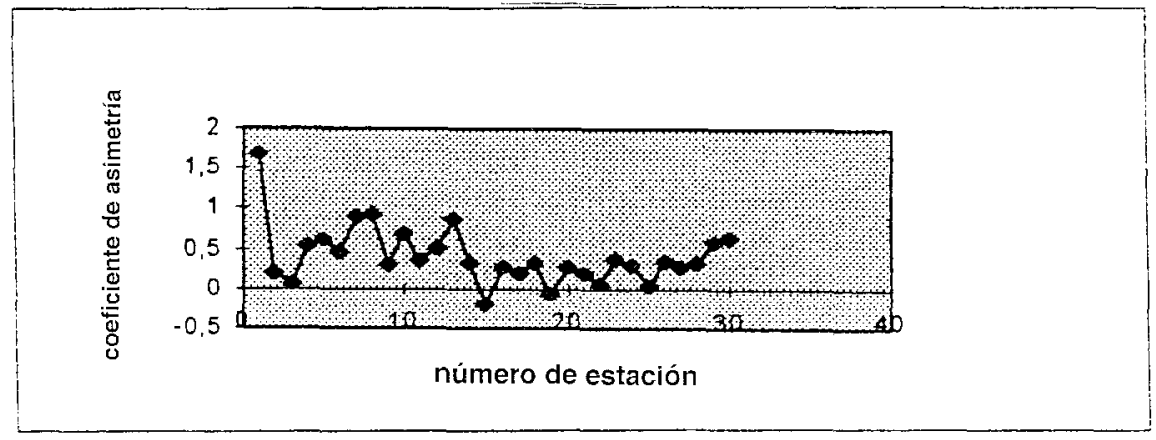

Grafico 3.- Curva de variación del Cs.

\section{IDENTIFICACIÓN DE LAS SUBCUENCAS HIDROLÓGICAMENTE HOMOGÉNEAS}

Se sigue el mismo procedimiento en cuanto al tratamiento de las aportaciones medias anuales y los caudales máximos díarios. La aplicación se centrará sobre 8 estaciones de aforo de subcuencas: Sigüés e Isaba sobre el río Esca, Biniés y Zuriza sobre el río Veral, Javierregay sobre el río Subordán y finalmente Jaca, Canfranc y Cola de embalse sobre el río Aragón. 
Tabla 1.- Análisis estadístico de la precipitación media anual de 33 estaciones meteorológicas.

\begin{tabular}{|c|c|c|c|c|c|c|c|}
\hline ESTACION & CODKGO & NUM. OBSERV. & PRE. M. AN. (m) & DESV. EST. (s) & $C v=s / m$ & Cs & REGION \\
\hline Candanchu & M09195 & 45 & 1966,8 & 521,202 & 0,265 & 1,678 & 1 \\
\hline Canfranc & M09198 & 45 & 1799,5 & 387,757 & 0,215 & 0,206 & 1 \\
\hline Villanua CE & M09199A & 45 & 1488,1 & 302,532 & 0,203 & 0,079 & il \\
\hline Aratores Mol. & M09199D & 45 & 1132,9 & 191,477 & 0,169 & 0,541 & $H$ \\
\hline Bescos Garc. & M09200 & 45 & 1062,2 & 170,311 & 0,160 & 0,621 & 11 \\
\hline Castiello Jac & M0201 & 45 & 1060,5 & 235,965 & 0,223 & 0,472 & 11 \\
\hline Jaca & MO202 & 45 & 772,4 & 122,443 & 0,159 & 0,915 & II! \\
\hline Canias & $M 0204$ & 45 & 824,2 & 120,137 & 0.146 & 0,920 & 111 \\
\hline Aisa de Jaca & M0205 & 45 & 1072,0 & 167,915 & 0,157 & 0,309 & $\|$ \\
\hline Binacua & M09206E & 45 & 651,3 & 131,574 & 0,202 & 0,696 & III \\
\hline Hecho & M09207 & 45 & 1161,7 & 211,258 & 0,182 & 0,349 & III \\
\hline Aragues det $P$ & M09208 & 45 & 1196,6 & 180,868 & 0,151 & 0,527 & Il \\
\hline Embun & M09209 & 45 & 734,6 & 155,626 & 0,212 & 0,882 & III \\
\hline Javierragay & M09210 & 45 & 752,7 & 156,565 & 0,208 & 0,335 & III \\
\hline Bailo Puente R & MO9210E & 45 & 716,8 & 141,510 & 0.197 & $-0,183$ & $\|$ \\
\hline Bagues & M09211 & 45 & 739,4 & 143,256 & 0,194 & 0,280 & II! \\
\hline Bailo & MO9211E & 45 & 765.8 & 143,492 & 0,187 & 0,210 & III \\
\hline Anso & MO9212 & 45 & 1162,5 & 239,694 & 0,206 & 0,338 & II \\
\hline Villa real & MOg214 & 45 & 722,1 & 175,936 & 0,244 & $-0,051$ & III \\
\hline Artieda & M09215 & 45 & 725,2 & 138,339 & 0,191 & 0,284 & III \\
\hline Isaba Belagua & M09216 & 45 & 2023,3 & 355,192 & 0,176 & 0,213 & I \\
\hline isaba & $M 09218$ & 45 & 1159,3 & 233,084 & 0,201 & 0,079 & ll \\
\hline Salvatierra & Mo9219! & 45 & 899,4 & 236,013 & 0,262 & 0,379 & III \\
\hline Sigues & Mos220 & 45 & 724.4 & 137,788 & 0,190 & 0,319 & III \\
\hline Leyre Monast. & Mog222U & 45 & 790,1 & 237,655 & 0,267 & 0,325 & HIt \\
\hline Yesa Embalse & M09223 & 45 & 771,4 & 197,801 & 0,256 & 0,350 & 111 \\
\hline Esparza & Mo8237I & 45 & 1189,1 & 223,754 & 0,188 & 0,285 & II \\
\hline Navacues & $\mathrm{MO} 238 \mathrm{H}$ & 45 & 888,8 & 184,783 & 0,208 & 0,334 & III \\
\hline Urries & M09243 & 45 & 617,9 & 132,278 & 0.214 & 0,599 & III \\
\hline Bernues & MO9470E & 45 & 663,7 & 149,946 & 0,226 & 0,635 & |f| \\
\hline
\end{tabular}

La selección de estas estaciones está basada en su pertenencia a la red de 1 a Confederación Hidrográfica del Ebro y tambien en la buena correlación $(R=0,996)$ de la superficie drenada por los ríos y las aportaciones medias anuales (Gráfico 4). 
Del análisis de las estadísticas de los caudales anuales máximos de medias diarias (Tabla 2 y Gráfico 5), se destaca que la zona de interés puede ser dividida en cuatro grupos de subcuencas hidrológicamente homogéneas, que a continuación conviene someter al test de homogeneidad según la ley de Gumbel:

La subcuenca de la Cola del embalse de Yesa.

Las subcuencas de Biniés, Zuriza, Isaba y Canfranc.

La subcuenca de Sigüés.

Las subcuencas de Jaca y Javierregay.

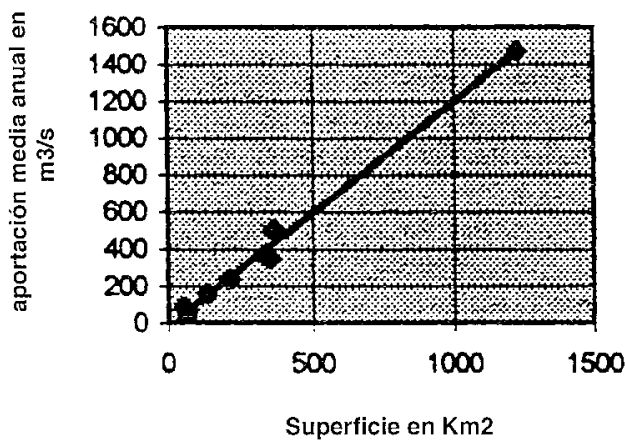

Grafico 4.- Aportación media anual y superficie.

Tabla 2.- Análisis estadístico de caudales máximos de medias diarias.

\begin{tabular}{|c|c|c|c|c|c|c|}
\hline N DE ESTACION & NUM.OBS. & C. M. AN. & DESV.ES. & $\mathrm{Cv}$ & Cs & REGION \\
\hline$N^{0} 18$ Jaca & 46 & 83,1 & 47,412 & 0,571 & 1,467 & IIII \\
\hline No 61 Javierragay. & 46 & 96,7 & 52,892 & 0,547 & 1,113 & III \\
\hline$N^{0} 62$ Blnies & 46 & 41,2 & 17,285 & 0,420 & 1,094 & IV \\
\hline$N^{\circ} 63$ Sigues & 46 & 141,1 & 53,888 & 0,382 & 0,522 & 11 \\
\hline No 170 Cola de Embalse & 46 & 306,7 & 160,643 & 0,524 & 1,037 & 1 \\
\hline$N^{0} 80$ Zuriza & 46 & 16,6 & 11,592 & 0,698 & 1,402 & IV \\
\hline$N^{0} 77$ lsaba & 46 & 32,3 & 16,219 & 0,502 & 0,919 & IV \\
\hline$N^{\circ} 164$ Canfranc & 46 & 29,2 & 13,322 & 0,456 & 1,513 & IV \\
\hline
\end{tabular}




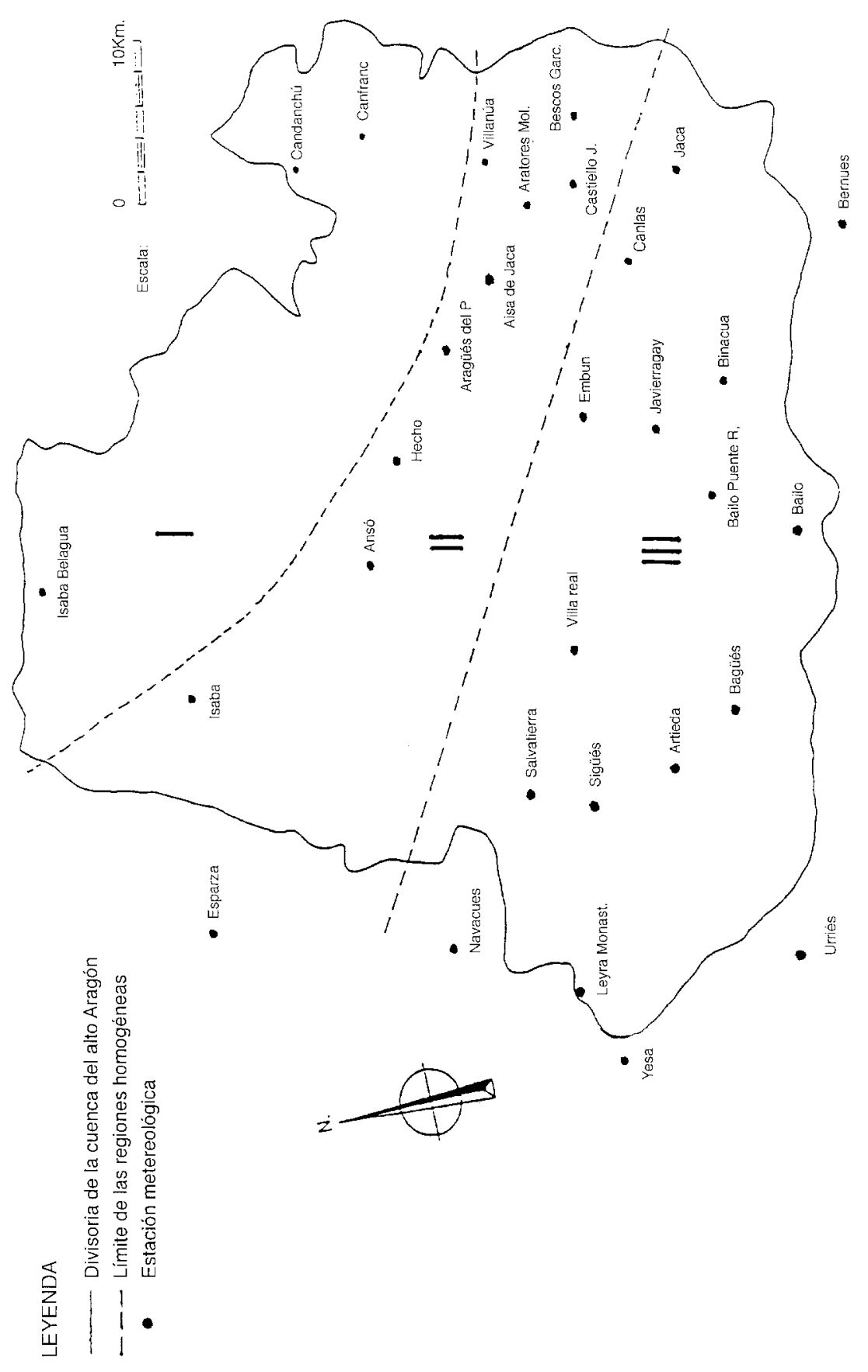

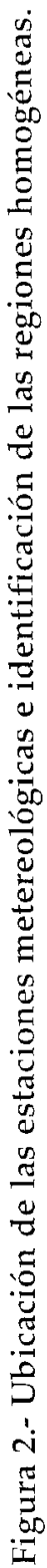




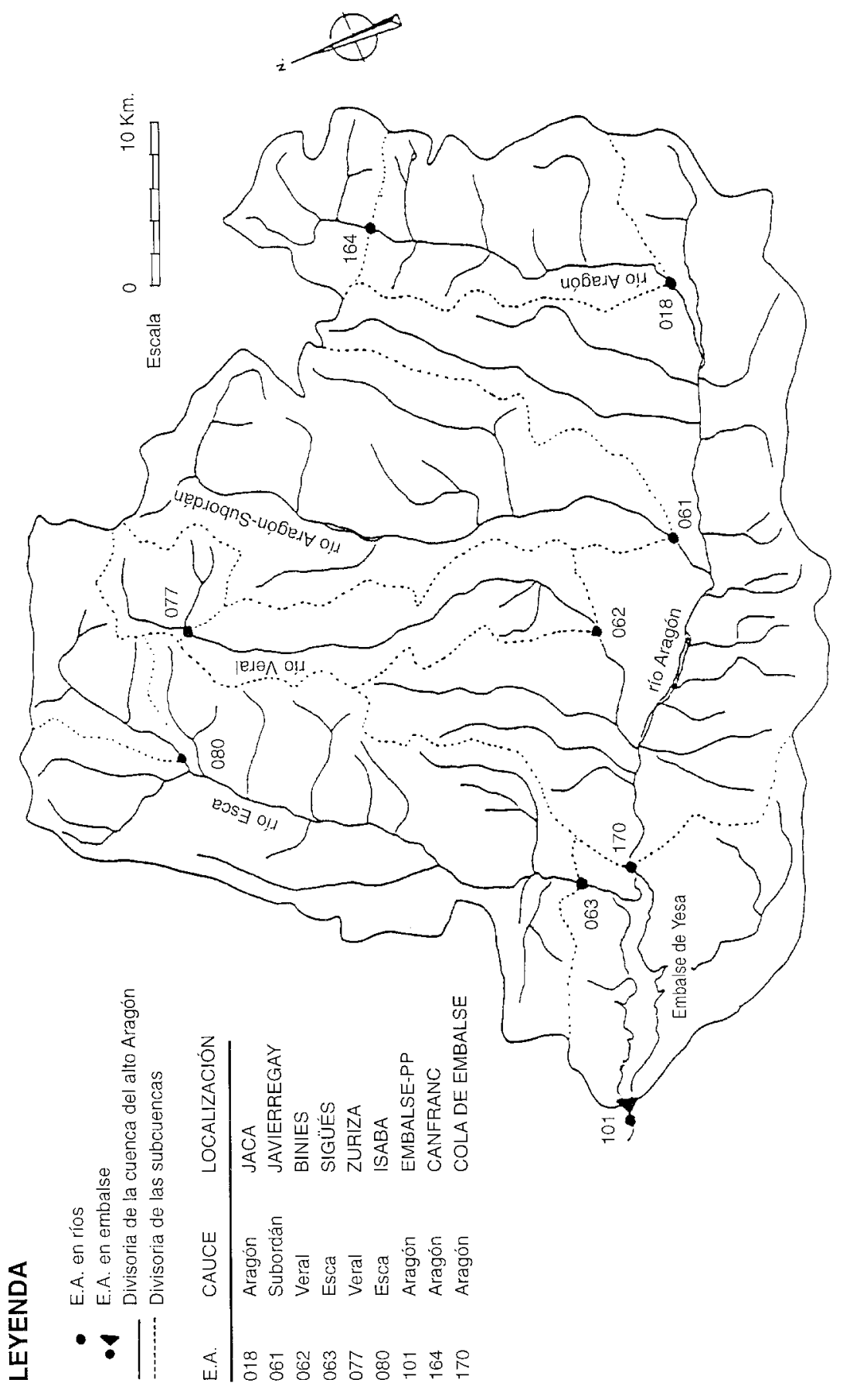

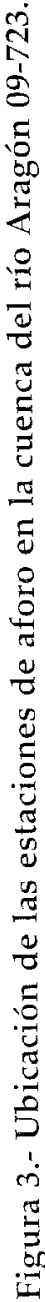




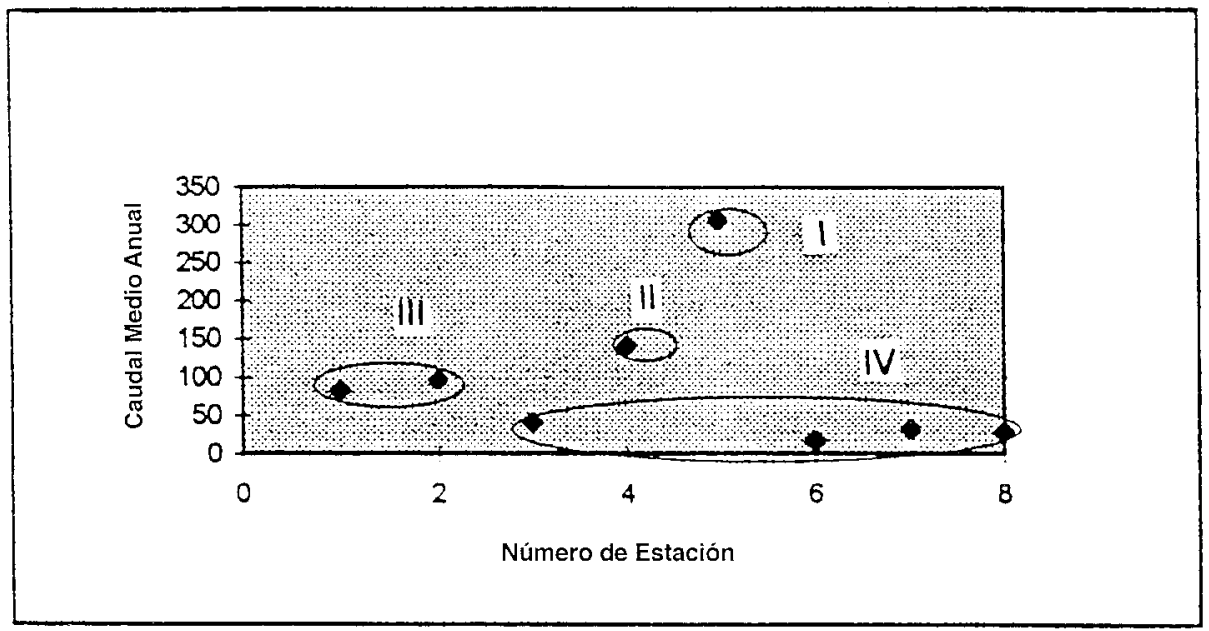

Grafico 5.- Identificación de subcuencas homogéneas.

\section{Teoría y aplicación del test de homogeneidad}

El test de homogeneidad fue desarrollado por Langbein, del Geological Survey de EEUU, a partir del principio siguiente: Se considera una muestra de 1000 observaciones de un fenómeno hidrológico, el caudal de un río, por ejemplo; si se divide esta muestra en 10 submuestras de 100 observaciones cada una y se calcula el caudal con un periodo de retorno $(T=10$ años) en cada una, puede esperarse que las variaciones de caudal de las 10 crecidas decenales sean pequeñas y fortuítas puesto que se trata, en definitiva, de una misma población. Decimos ahora que las 10 submuestras son homogéneas.

En nuestro caso, tenemos 8 estaciones con 45 observaciones de caudales máximos anuales. Es evidente que los 8 periodos de retorno T10 serán diferentes, pero habrá que saber si las variaciones son únicamente debidas al azar o bien al hecho de que las muestras no pertenecen a la misma población. De otro modo, se plantea saber si las 8 muestras son homogéneas y, por eso, hay que cuantificar los límites de las variaciones admisibles, fuera de las cuales se rechaza la hipótesis de homogeneidad.

Según la ley de Gumbel el test puede demostrar que aproximadamente el $65 \%$ de los periodos de retorno Tr estimados están dentro de una banda de ancho dy el $95 \%$ de Tr estimados están en una banda de ancho $2 y$. Generalmente este tipo de test se hace para periodos de retorno $\operatorname{Tr}=10$ años. 
Sobre papel de probabilidades de valores extremos (papel de Gumbel) se trazan, para cada estación, los caudales anuales en función de Tr. Después de ajustar las rectas de frecuencias se calcula gráficamente el módulo interanual Q2,33 y el caudal de periodo de retorno decenal Q10.

El factor de uniformidad es :

$$
\mathrm{K}=((\mathrm{Q} 10 / \mathrm{Q} 2,33) / \mathrm{m}
$$

Y para cada estación, el caudal uniformizado es:

$$
\mathrm{Qk}=\mathrm{KQ2,33}
$$

En las curvas de distribución correspondientes, se calculan los distintos periodos de retorno Trk y los conjuntos (Trk, n) se llevan sobre el papel de probabilidad donde previamente se trazaron las bandas de confianza de $95 \%$ y de $65 \%$.

Las Tablas 3, 4 y 5, y los Gráfico 6 a y 6 b muestran los resultados obtenidos.

Solo las estaciones de Javierregay, Cola de embalse, Isaba, Zuriza y Canfranc se encuentran en la región de aceptación del test, dentro de las bandas de confianza de $95 \%$, mientras que las bandas de confianza de $65 \%$ rechazan Zuriza y Canfranc.

\begin{tabular}{|c|c|c|c|c|c|c|}
\hline N DE ESTACION & $\mathrm{Q} 2,33(\mathrm{~m} 3 / \mathrm{s})$ & Q10 (m3/s) & Q10/02,33 & $Q_{K}=K K_{2,33}$ & $\operatorname{Tr}(a n c s)$ & n (anos) \\
\hline$N^{2} 18$ Jaca & 85,0 & 146,1 & 1,719 & 237,9 & 40.33 & 39 \\
\hline N 61 Javiertagay. & 54,2 & 183,2 & 3,380 & 151.7 & 6,00 & 24 \\
\hline$N^{*} 62$ Binios & 27,9 & 62,0 & 2.202 & 78,1 & 16,34 & 39 \\
\hline N 60 Sigües & 101,9 & 218,4 & 2,143 & 285,2 & 66,67 & 38 \\
\hline $\mathrm{N}^{\circ} 170$ Cota de Em. & 177,5 & 529,4 & 2,960 & 496,8 & 9,35 & 11 \\
\hline$N^{*} 80$ Zuriza & 8,3 & 38,8 & 4,675 & 23.2 & 5,21 & 30 \\
\hline$N^{*} 77$ Isaba & 19.1 & 52,4 & 2,744 & 53.5 & $9, \infty$ & 18 \\
\hline No 164 Cantranc & 19,4 & 49.0 & 2.526 & 54.3 & 23.04 & 19 \\
\hline
\end{tabular}

Tabla 3.- Cálculos de las pruebas de homogeneidad. 
Tabla 4.- Test de homogeneidad - Valores de $\operatorname{Tr}$ (bandas de confianza $a=0,05$ ) $\mathrm{y}^{10}$ : estimación de la media $=2,25$.

\begin{tabular}{|c|c|c|c|c|c|}
\hline \multirow{2}{*}{$\begin{array}{c}n \\
\text { (años) }\end{array}$} & \multirow{2}{*}{$\begin{array}{c}2 a= \\
6,33 / \text { no.5 }\end{array}$} & \multicolumn{2}{|l|}{ límite inferior } & \multicolumn{2}{|l|}{ limite superior } \\
\hline & & $y_{10}-2 a$ & $\mathrm{Ti}$ & $y_{10}+2 a$ & $T_{s}$ \\
\hline 5 & 2,84 & 0,59 & 1.20 & 5,09 & 160.0 \\
\hline 10 & 200 & 0,25 & 1.85 & 4,25 & 70,0 \\
\hline 20 & 1,42 & 0,83 & 2.80 & 3.67 & 40,0 \\
\hline 30 & 1,16 & 1,09 & 3,50 & 3,41 & 30,0 \\
\hline 40 & $1, \infty$ & 1.25 & 4,05 & 3.25 & 26,0 \\
\hline 50 & 0,90 & 1,35 & 4,40 & 3,15 & 24,0 \\
\hline 100 & 0,63 & 1,62 & 5,60 & 2,88 & 18,0 \\
\hline 200 & 0.45 & 1,80 & 6,50 & 2,70 & 15,5 \\
\hline 500 & 0,28 & 1,97 & 7,70 & 2,53 & 13,0 \\
\hline 1000 & 0,20 & 2,05 & 8,30 & 2,45 & 12.0 \\
\hline
\end{tabular}

Tabla 5.- Test de homogeneidad - Valores de $\operatorname{Tr}$ (bandas de confianza $a=0,35$ ) $\mathrm{y}^{10}$ : estimación de la media $=2,25$

\begin{tabular}{|c|c|c|c|c|c|}
\hline \multirow{2}{*}{$\begin{array}{c}n \\
\text { (ah⿱os) }\end{array}$} & \multirow{2}{*}{$\begin{array}{c}a= \\
6,33 / n 0,5\end{array}$} & \multicolumn{2}{|l|}{ limite inferior } & \multicolumn{2}{|l|}{ limite superior } \\
\hline & & $y_{10}-a$ & $\mathrm{Ti}$ & y10 +a & $T_{s}$ \\
\hline & 1,42 & 0,83 & 2,83 & 3,67 & 39,75 \\
\hline & $1, \infty$ & 1,25 & 4,01 & 3,25 & 26,29 \\
\hline & 0,71 & 1,54 & 5,18 & 2,96 & 19,80 \\
\hline & 0,58 & 1,67 & 5,83 & 2,83 & 17,45 \\
\hline & 0,50 & 1,75 & 6,27 & 2,75 & 16,15 \\
\hline & 0,45 & 1,80 & 6,57 & 2,70 & 15,39 \\
\hline & 0,32 & 1,93 & 7,40 & 2,57 & 13,57 \\
\hline & 0,23 & 2,02 & 8,05 & 2,48 & 12,45 \\
\hline & 0.14 & 2,11 & 8,76 & 2,39 & 19,42 \\
\hline
\end{tabular}

\section{IDENTIFICACIÓN DE LA SUBCUENCA CLIMÁTICA E HIDROLÓGICAMENTE HOMOGÉNEA}

Teniendo en cuenta que en este estudio se propone seleccionar una subcuenca climática y hidrológicamente homogénea, con una extensión que trata de abarcar de Norte a Sur las tres regiones homogéneas identificadas, la ilustración de las curvas de homogeneidad demuestra que sólo las estaciones de Javierregay, Cola de embalse e Isaba se encuentran en la región de aceptación de las curvas de confianza de $65 \%$. Finalmente nos quedan dos subcuencas, la cola de embalse y Javierregay de las cuales tenemos que elegir la más adecuada para los trabajos de campo (la estación de Isaba ha sido descartada por su corta extensión geográfica). 


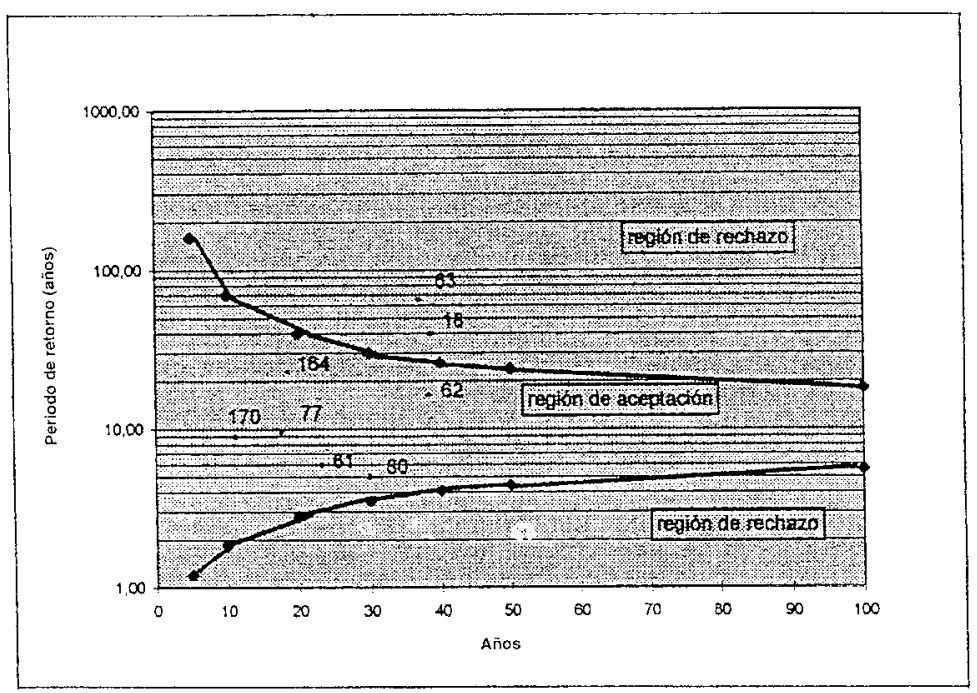

Gráfico 6a.- Test de homogeneidad, bandas de confianza $(a=0.05)$. Los números hacen referencia a las estaciones de aforo.

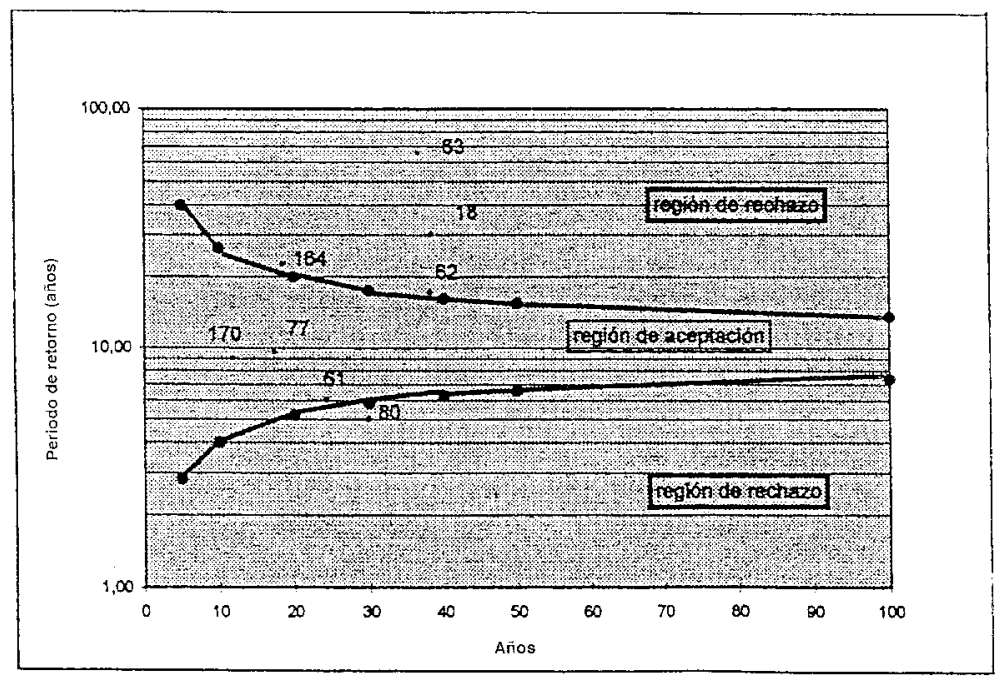

Gráfico 6b.- Test de homogeneidad, bandas de confianza (a=0.35). Los números hacen referencia a las estaciones de aforo. 
Respecto a la varibilidad de los coeficientes de correlación y de sesgo de ambas estaciones, no se destacan realmente diferencias muy significativas.

Estación

Javierregay

Cola de embalse
$\mathrm{CV}$

0,547

0,524
Cs

\section{1,113}

1,017

En el Gráfico 7 se ha trazado la recta de regresión entre los módulos interanuales Q2,33 y las superficies de las subcuencas correspondientes, lo que es un enfoque interesante para mejor esclarecer el análisis entre las estaciones de Javierregay y Cola de embalse. La ecuación de regresión es:

$$
\mathrm{Q} 2,33=0,1142 \mathrm{~A}+20 \text {, donde: }
$$

Q2,33 es el módulo interanual, y

A es la superficie de la cuenca en $\mathrm{km}^{2}$.

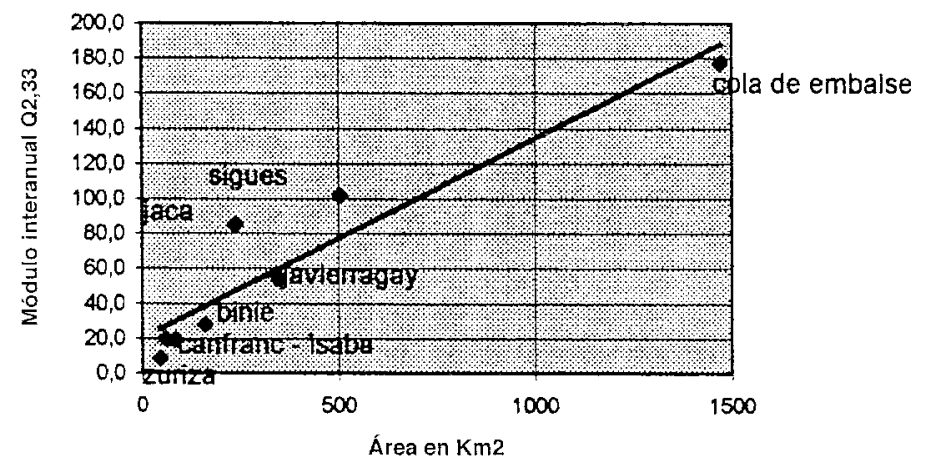

Gráfico 7.- Recta de regresión.

\section{DISCUSIÓN}

$1^{\circ}$.- En relación con la identificación de las $\mathrm{RCH}$, la estación de Belagua ha sido incluida en la región (I) a causa de su ubicación geografica aunque tiene un alto valor de precipitación media anual (PMA $=2023,3 \mathrm{~mm}$ ), respecto las otras estaciones de 1 a región. También destaca una significativa variabilidad del coeficiente de sesgo (Cs = $1,678)$ que corresponde a la misma estación de Belagua, pero la variabilidad del coeficiente de variación $(0,176 ; 0,215 ; 0265)$ de las tres respectivas estaciones de Belagua, Canfranc y Candanchú, favorece la conservación del grupo (I). 
De otra parte, la estación de Villanúa ha sido incorporada a la zona (II), a pesar de que tiene un valor de PMA sensiblemente alto $(1488,1 \mathrm{~mm})$ que el resto de su grupo, porque los coeficientes de variación que les corresponden se aproximan a los $\mathrm{CV}$ de su región.

La precipitación media anual cumulativa, el $\mathrm{Cv}$ medio y el Cs medio de las regiones I, II y III son respectivamente: $(1929,9 ; 1169,2$ y 773,4); $(0,219 ; 0,165 ; 0,208)$; $(0,699 ; 0,361 ; 0,421)$.

$2^{\circ}$.- Respecto a la identificación de las ScHH, se destaca del gráfico de las bandas de confianza calculadas y que definen la región de aceptación o rechazo de la hipótesis de homogeneidad, lo siguiente:

- En las bandas de confianza de $95 \%$ se han clasificado las subcuencas de Jaca, Sigüés y Biniés en la región de rechazo, y se han considerado como homogĖneas las subcuencas de Javierregay, Zuriza, Isaba, Canfranc y Cola de embalse.

- A las bandas de confianza de 65\%, sólo las subcuencas de Javierregay, Cola de embalse y Isaba quedan dentro de estas bandas, el resto de las muestras a que añadimos Isaba (por su corta extensión geográfica), están descartadas como subcuencas homogéneas.

$3^{\circ}$.- Comparando los coeficientes de variación y de sesgo de los datos de aportaciones medias anuales y de caudales máximos díarios de ambas estaciones de aforo (Javierregay y Cola de embalse), se observa que no presentan diferencias muy significativas. Por tanto, parece juicioso adoptar la relación Superficie-Módulo interanual, para poder concretar lo más posible la subcuenca climática e hidrológicamente homogénea.

El gráfico 7 muestra que la estación de Javierregay se representa muy bien en 1 a recta de regresión lineal. Además, se nota que las diferencias entre el módulointeranual calculado y el determinado mediante la ecuación de regresión reflejan los siguientes valores:

- A Javierregay le corresponde la diferencia de $5,5406 \mathrm{~m}^{3} / \mathrm{s}$.

- En. cuanto a la Subcuenca de la Cola de Embalse, se estima una diferencia de $12,4872 \mathrm{~m}^{3} / \mathrm{s}$.

Esta última comparación demuestra que la subcuenca de Javierregay tiene más ventaja que la de Cola de embalse; por tanto, la cuenca drenada por el río Subordán puede ser adoptada como subcuenca climática e hidrológicamente homogénea. 


\section{CONCLUSIÓN}

El trabajo presentado realiza una selección de una subcuenca climática e hidrológicamente homogénea dentro de una extensa región. El empleo de un método de análisis estadístico regional de parámetros, utilizando los datos de precipitación media anual y datos hidrométricos, ha permetido alcanzar el objetivo planteado.

La delimitación de tres regiones geográficas, cuya identificación ha sido determinada mediante las técnicas estadísticas y la ubicación geográfica, ha permitido destacar unas pequeñas diferencias de los parámetros estimados, lo que en cierto modo dificulta incluir tal estación climática en la región homogénea que le corresponde. Por eso, contar con dos o más parámetros permitirá reforzar la capacidad descriptiva del método adoptado.

La comparación de los resultados de la aplicación del test de homogeneidad de Gumbel con los datos hidrométricos ha permitido concretar las subcuencas hidrológicamente homogéneas. Además, la ilustración de la relación superficiemódulo interanual, constituye un enfoque conveniente para seleccionar, y de manera acusable, la subcuenca homogénea requerida.

Del análisis de los Cv y Cs se destaca que la subcuenca de Javierregay tiene más ventaja que la de Cola de embalse. También, en el Gráfico 7 se observa que la estación de Javierregay se representa muy bien en la recta de regresión, mejor que el resto de las subcuencas. En consecuencia, parece que la subcuenca drenada por el río Subordan puede ser considerada como región homogenea adecuada para llevar a cabo los trabajos de campo. Por tanto, se adoptará como subcuenca climática e hidrológicamente homogénea.

\section{BIBLIOGRAFÍA}

NAGHAVI, B. \& FANG X.Y. (1995): "Regional Frequency Analisis of Extreme Precipitation in Luisiana". Journal of Hydraulic Engineering, 819-824.

CAMPOS, D.F. (1994): "Aplicación del Método del Indice de Crecientes en la Región Hidrológica Número 10, Sinaloa". Ingeniería Hidráullica en México, vol. IX, 3, 41-55.

FRANCES, F. (1991): Utilización de la información histórica en el Análisis Regional de las Avenidas. Tésis Doctoral. Universidad Politécnica de Valencia.

FERRER, J. y ARDILES, L. (1994): Análisis Estadístico de las Series Anuạles de Máximas Lluvias Diarias en España. Centro de Estudios Hidrográficos (MOPT). 
LLAMAS, J. (1993): Hidrología General, Principios y Aplicaciones. Servicio Editorial de la Universidad del Pais Vasco.

W.M.O. (1989): "Statistical Distributionsfor Flood Frequency Análisis". World Meteorological Organisation. Operational Hyd:logy Repor! 33, 73 p. 